\section{Incidence and recognition of acute respiratory distress syndrome in a UK intensive care unit}

\section{ABSTRACT}

The reported incidence of ARDS is highly variable $(2.5 \%-19 \%$ of intensive care unit (ICU) patients) and varies depending on study patient population used. We undertook a 6-month, prospective study to determine the incidence and outcome of ARDS in a UK adult University Hospital ICU. 344 patients were admitted during the study period, of these 43 (12.5\%) were determined to have ARDS. Patients with ARDS had increased mortality at 28 days and 2 years post-diagnosis, and there was under-recognition of ARDS in both medical records and death certificattion. Our findings have implications for critical care resource planning.

The acute respiratory distress syndrome (ARDS) is characterised by pulmonary inflammation and increased pulmonary vascular permeability, which results in non-cardiogenic pulmonary oedema and refractory hypoxaemia. ${ }^{1}$ ARDS is associated with significant morbidity and mortality, and increased usage of critical care resources. The reported incidence of ARDS is highly variable: ARDS has been reported in $2.5 \%-19 \%$ of intensive care unit (ICU) patients. ${ }^{2-8}$ Aside from an audit conducted in 23 Scottish ICUs in 2003, which identified an ARDS incidence of $8.1 \%,{ }^{6}$ there have been no recent studies in the UK. Reported mortality rates for ARDS also vary substantially, with values for inhospital mortality ranging from $23 \%$ to $72 \% .^{9}$ To address this we undertook a 6-month, prospective study to determine the incidence and outcome of ARDS in a UK adult University Hospital ICU.

Our study was approved by Cambridgeshire 3 Ethics Committee (08/ $\mathrm{H} 0306 / 17)$ and conducted in the John V Farman (general adult) ICU at Cambridge University Hospitals NHS Foundation Trust; a 20-bed ICU, serving a 1250-bed University Hospital with a local catchment of 350000 , and a wider regional referral base of $\sim 2$ million people. A dedicated clinical research team, not involved in patient care, prospectively studied all patients admitted during a 6-month period (1 January-30 June 2009). The ICU clinical staff were not aware of the aim of the data collection. Data were collected daily until patient discharge from ICU; patients were then followed up to determine their date of hospital discharge, and survival status at 28 days, 6 months and 2 years.

All patients with a P:F ratio of $<40 \mathrm{kPa}$ on two arterial blood gases, taken at least 6 hours apart, and bilateral opacities on thoracic radiography had their medical notes, hospital investigations and chest radiographs reviewed by a physician who was independent of the data collection to confirm or exclude the diagnosis of ARDS. ${ }^{10}$ All other patients were considered not to have ARDS ('No Lung Injury', NLI). Assessment of left atrial hypertension was based on objective criteria when available. Patients were excluded if they had any of the following: biochemical or electrical evidence of an acute myocardial infarction, previous or current echocardiograms showing moderate or greater dilated left atrium or left ventricular dysfunction or an enlarged cardiac silhouette on a recent posterioranterior chest radiograph. A randomly generated sample of $40 \%$ of patients with NLI had their cases reviewed in the same manner as above, to confirm the absence of ARDS. Only one case initially classified as NLI was reclassified as ARDS. For patients with a confirmed diagnosis of ARDS, copies of their hospital discharge summaries and medical coding were obtained, and the medical death certificates of those patients who died in hospital were reviewed.

Data were analysed using GraphPad Prism. Categorical variables were described using proportions and analysed using Fisher exact or $\chi^{2}$ tests. Continuous variables were described using median (IQR), and analysed using Mann-Whitney test as they were found not to follow a Gaussian distribution. Survival curves were compared using Mantel-Cox test.
For the purposes of calculating length of ICU/hospital stay, the date of death was assumed to be the date of discharge from ICU/hospital. A p value of $<0.05$ was considered significant.

Three hundred and forty-four patients were admitted during the study period, of these $43(12.5 \%)$ were determined to have ARDS (table 1). Our data are consistent with the previously cited Scottish study, and a multicentre European study from 2002, ${ }^{6} 7$ suggesting that the UK may not have observed the same decline in ARDS incidence reported in US study populations. $^{11}$

There was no difference in age (median 61 vs 63 years) or sex (males: $51 \%$ vs $56.8 \%$ ) between patients who had ARDS and those who did not. The Acute Physiology and Chronic Health Evaluation (APACHE) II score on admission to ICU was significantly greater for patients who had or subsequently developed ARDS, compared with patients who did not (median 20 (17-24) vs 16 (1321); p <0.0001). Pulmonary sepsis was the most common cause of ARDS, occurring in 29 out of 43 cases (67.4\%). Nine cases of ARDS were associated with nonpulmonary sepsis, four cases were transfusion-associated acute lung injury (TRALI) and one occurred in the setting of polytrauma. The majority of the ARDS patient cohort $(62.7 \% ; n=27)$ met ARDS diagnostic criteria at the time of their admission to ICU.

Patients with ARDS had significantly fewer ventilator-free days within the first 28 days following ICU admission (median 7 (0-14) vs 27 (25-28) days; $\mathrm{p}<0.0001)$, and longer ICU stays than those who did not develop ARDS (median 15 (4-23) vs 3 (2-6.5) days; $\mathrm{p}<0.0001)$. All study

Table 1 Incidence and outcome of ARDS in UK teaching hospital ICU

\begin{tabular}{lccc}
\hline & NLI & ARDS & p Value \\
\hline $\mathrm{n}(\%)$ & $301(87.5)$ & $43(12.5)$ & \\
Median age in years (IQR) & $63(49-75)$ & $61(45-75)$ & 0.7690 \\
Male, $\mathrm{n}(\%)$ & $171(56.8 \%)$ & $22(51.1 \%)$ & 0.5141 \\
Median APACHE II score on admission to ICU (IQR) & $16(13-21)$ & $20(17-24)$ & $<0.0001$ \\
Median length of hospital stay prior to ICU admission in days (IQR) & $2(1-7)$ & $3(2-18)$ & $<0.001$ \\
Median length of ICU stay in days (IQR) & $3(2-6.5)$ & $15(4-23)$ & $<0.0001$ \\
Median length of hospital stay in days (IQR) & $23(11-43)$ & $38(13-69)$ & 0.0502 \\
Mortality in ICU, $\mathrm{n}(\%)$ & $34(11.3 \%)$ & $18(41.9 \%)$ & $<0.0001$ \\
Mortality in hospital, $\mathrm{n}(\%)$ & $64(21.2 \%)$ & $23(53.5 \%)$ & $<0.0001$ \\
Mortality at 28 days, $\mathrm{n}(\%)$ & $46(15.3 \%)$ & $17(39.5 \%)$ & $<0.0001$ \\
Mortality at 6 months, $\mathrm{n}(\%)$ & $73(24.3 \%)$ & $24(55.8 \%)$ & $<0.0001$ \\
Mortality at 2 years, $\mathrm{n}(\%)$ & $108(35.9 \%)$ & $27(62.8 \%)$ & $<0.0001$ \\
\hline APACHE, Acute Physiology and Chronic Health Evaluation; ARDS, acute respiratory distress syndrome; ICU, intensive care \\
unit; NLI, no lung injury. & & &
\end{tabular}




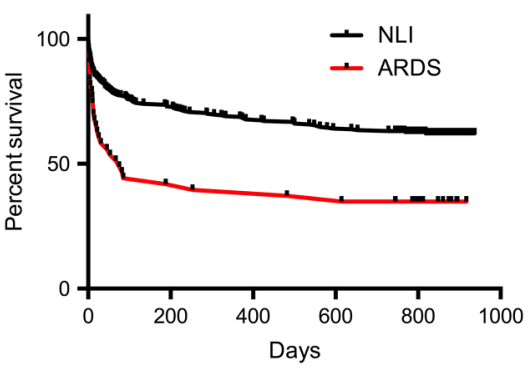

Figure 1 Kaplan-Meier survival curves for patients with acute respiratory distress syndrome (ARDS) or no lung injury (NLI) groups.

patients received standard mechanical ventilation (ie, none received extracorporeal support, high frequency oscillation or other advanced respiratory support modalities). There was an increased length of hospital stay (median 38 (13-69) vs 23 (11-43) days; $p=0.05)$ in patients with ARDS, but this was not significant at the $5 \%$ level.

Patients with ARDS had ICU and hospital mortality rates of $41.9 \%$ and $53.5 \%$, respectively; these were significantly greater than the ICU and hospital mortality rates for patients with NLI of $11 \%$ and $21 \%$, respectively $(\mathrm{p}<0.001, \mathrm{p}<0.05)$. Figure 1 shows the Kaplan-Meier survival curves for each patient group. Patients with ARDS have a significantly reduced longterm survival compared with patients who had NLI $(\mathrm{p}<0.0001)$.

Despite a requirement that all patients, including those who die during their admission, admitted to our hospital should receive a discharge summary, of the 43 patients with ARDS, only 20 had a completed electronic hospital discharge summary. Remarkably, of these 20, only one made reference to the development of ARDS during the admission. Again, of the patients that died prior to hospital discharge, only one had a medical death certificate where ARDS was specified as a contributing cause of death, with many others $(48 \%)$ recording 'multiple organ failure' as a cause of death. The lack of documentation on medical death certificates may reflect that patients often die of non-pulmonary organ failure, rather than directly from ARDS. Of the 43 patients with ARDS, only two were coded as such by the hospital's coding department.

The under-recognition of ARDS by both the clinical and coding teams, and the omission of any reference to ARDS in the medical correspondence may have wide ranging implications in terms of the immediate management for individual patients (eg, timely commencement of lung protective ventilation and other supportive measures), and in their follow-up care. Long-term follow-up studies have shown that ARDS survivors suffer major and ongoing reductions in their health/ functional status. ${ }^{12}$ Under-recognition also has implications for resource planning with regard to ensuring critical care facilities meet demands, for example, the provision of extracorporeal membrane oxygenation services.

A limitation of this study is the potential for misclassification of ARDS, due in part to the subjective nature of the diagnostic criteria. However, to minimise this, a physician independent of the data collection team reviewed all potential cases of ARDS, as well as a representative $40 \%$ sample of patients who did not. Further weaknesses of our study include it being a single centre study, the potential for the study nurse involved in the prospective data collection to influence the outcome, and the low incidence of ARDS due to non-septic causes. Despite this, our study highlights the impact of ARDS on patient outcomes and ICU/hospital resource usage.

\section{Charlotte Summers, ${ }^{1,2}$ Nanak R Singh, ${ }^{1}$ Linda Worpole, ${ }^{1}$ Rosalind Simmonds, ${ }^{1}$ Judith Babar, ${ }^{3}$ Alison M Condliffe, Kevin E Gunning, ${ }^{2}$ Andrew J Johnston, ${ }^{2}$ Edwin R Chilvers}

'Department of Medicine, University of Cambridge School of Clinical Medicine, Cambridge, UK ${ }^{2} J o h n$ V Farman Intensive Care Unit, Addenbrooke's Hospital, Cambridge University Hospitals NHS Foundation Trust, Cambridge, UK

${ }^{3}$ Department of Radiology, University of Cambridge School of Clinical Medicine, Cambridge, UK

Correspondence to Dr Charlotte Summers, Division of Anaesthesia, Department of Medicine, Box 93, Level 4, Addenbrooke's Hospital, CUHNHSFT, Hills Road, Cambridge CB2 OQQ, UK; cs493@medschl.cam.ac.uk CS and NRS contributed equally.

Acknowledgements We are grateful to the staff of the John V Farman Intensive Care Unit, Addenbrooke's Hospital, Cambridge, UK for cooperating with this work.

Contributors CS, ERC, AMC, KEG and AJJ contributed to the concept/design of the study. LW and RS collected the data. NRS, JB and ERC undertook the data validation. CS analysed the data. CS and ERC wrote the manuscript.

Funding The study was supported by the Wellcome Trust (WT101692MA) (CS). Intensive Care Society; NIHR Cambridge Biomedical Research Centre, Papworth Hospital and East of England CRN.

Competing interests CS, AMC and ERC have received project grant funding from GSK to undertake preclinical studies of the effect of novel therapeutic agents relevant to ARDS. The authors have no other relevant interest to declare.

Ethics approval Cambridgeshire 3 REC.

Provenance and peer review Not commissioned; externally peer reviewed.

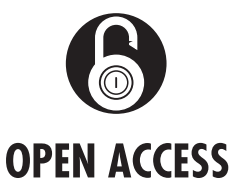

Open Access This is an Open Access article distributed in accordance with the terms of the Creative Commons Attribution (CC BY 4.0) license, which permits others to distribute, remix, adapt and build upon this work, for commercial use, provided the original work is properly cited. See: http:/l creativecommons.org/licenses/by/4.0/

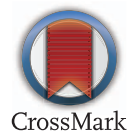

To cite Summers C, Singh NR, Worpole L, et al. Thorax 2016;71:1050-1051.

Received 26 January 2016

Revised 18 April 2016

Accepted 9 May 2016

Published Online First 22 August 2016

Thorax 2016;71:1050-1051.

doi:10.1136/thoraxjnl-2016-208402

\section{REFERENCES}

1 Matthay MA, Ware LB, Zimmerman GA. The acute respiratory distress syndrome. J Clin Invest 2012;122:2731-40.

2 Webster NR, Cohen AT, Nunn JF. Adult respiratory distress syndrome- how many cases in the UK? Anaesthesia 1998;43:923-6.

3 Fialkow L, Vieira SRR, Fernandes AK, et al. Acute Respiratory Distress Syndrome Research Group. Acute lung injury and acute respiratory distress syndrome at the intensive care unit of a general university hospital in Brazil. An epidemiological study using the American-European Consensus Criteria. Intensive Care Med 2002;28:1644-8.

4 Brun-Buisson C, Minelli C, Bertolini G, et al. Epidemiology and outcome of acute lung injury in European intensive care units. Results from the ALIVE study. Intensive Care Med 2004;30:51-61.

5 Bersten AD, Edibam C, Hunt T, et al. Incidence and mortality of acute lung injury and the acute respiratory distress syndrome in three Australian States. Am J Respir Crit Care Med 2002;165:443-8.

6 Hughes M, MacKirdy FN, Ross J, et al. Acute respiratory distress syndrome: an audit of incidence and outcome in Scottish intensive care units. Anaesthesia 2003:58:838-45.

7 Vincent JL, Sakr Y, Groeneveld J, et al. ARDS of early or late onset: does it make a difference? Chest 2010;137:81-7.

8 Irish Critical Care Trials Group. Acute lung injury and the acute respiratory distress syndrome in Ireland: a prospective audit of epidemiology and management. Crit Care 2008;12:R30.

9 Zambon M, Vincent JL. Mortality rates for patients with acute lung injury/ARDS have decreased over time. Chest 2008;133:1120-7.

10 Ranieri VM, Rubenfeld GD, Thompson BT, The ARDS Definition Task Force. Acute respiratory distress syndrome: the Berlin definition. JAMA 2012;307:2526-33.

11 Li G, Malinchoc M, Cartin-Ceba R, et al. Eight-year trend of acute respiratory distress syndrome: a population-based study in Olmsted County, Minnesota. Am J Respir Crit Care Med 2011;183:59-66.

12 Herridge MS, Tansey CM, Matté A, et al. Functional disability 5 years after acute respiratory distress syndrome. N Engl J Med 2011;364:1293-304. 